\title{
COMMENT
}

\section{Preserving some sanity}

\author{
Gregory A Petsko*
}

A popular definition of insanity - frequently misattributed to Albert Einstein or Benjamin Franklin, but probably originating with novelist Rita Mae Brown in 1983 - is that insanity is doing the same thing over and over again but expecting a different outcome. If that definition is a good one, then I think there is a possibility that some of the people who run American science might want to start getting fitted for a new jacket - the kind where the sleeves wrap around the front and are tied together in back.

Before I explain why I think that could happen, it's worthwhile going over some of the history of scientific funding in the United States in the past 40 years, to see how we got ourselves into the situation we're in now. In 1971, the US President, Richard Nixon announced a War on Cancer. In his State of the Union address in January of that year, he proclaimed: "I will also ask for an appropriation of an extra $\$ 100$ million to launch an intensive campaign to find a cure for cancer, and I will ask later for whatever additional funds can effectively be used. The time has come in America when the same kind of concentrated effort that split the atom and took man to the moon should be turned toward conquering this dread disease. Let us make a total national commitment to achieve this goal." On December 23 that same year, he signed the National Cancer Act into law, declaring, "I hope in the years ahead we will look back on this action today as the most significant action taken during my Administration."

Well, in retrospect, I think we can all agree that the Watergate cover-up probably turned out to be the most significant action of his Administration, but this one was right up there. The National Cancer Act (P.L. 92-218), "The War on Cancer," gave the National Cancer Institute, one of the institutes at the National Institute of Health (NIH), unique autonomy at NIH with special budgetary authority. Over the last four decades it has grown into, by far, the largest of the 27 Institutes and Centers that make up the biggest biomedical research funding agency in the world. Its annual budget is now just a little under $\$ 5$ billion (out of a total NIH budget of about $\$ 32$ billion) and it is still

*Correspondence: petsko@brandeis.edu

Rosenstiel Basic Medical Sciences Research Center, Brandeis University, Waltham, MA 02454-9110, USA charged with coordinating the National Cancer Program in other words, the War on Cancer goes on. Now, certainly there have been many victories in that war: testicular cancer is no longer a fatal disease thanks to Barnett Rosenberg's discovery of cisplatinum as an anticancer agent; chronic myelogenous leukemia is now treatable by Gleevec and other Bcr-Abl kinase inhibitors thanks to the discovery by David Baltimore and others that survival of that tumor depends on that kinase and the efforts of Brian Drucker and Nick Lydon to exploit that discovery; Her-2 positive breast cancer is treatable by antibodies directed at that cell surface protein thanks to the work of Dennis Slamon; other forms of breast cancer can now be attacked by aromatase inhibitors thanks to the work of Angela Hartley Brodie; multiple myeloma now has a treatment thanks to the work of Fred Goldberg, who proposed the seemingly insane idea that inhibiting the proteasome might be beneficial and not all that toxic; and I could give many more examples - the list is a long one and the victories are impressive indeed. But in most of these cases the key work, the initial discovery that led to the treatment, was not funded as part of the War on Cancer and was in many cases - cisplatinum being the greatest example - not even done with curing a disease in mind. And of course, most cancers, especially solid tumors, are still very hard to treat and are often fatal. We've won many battles, but Congress and the public will be forgiven for asking: just how long is this war going to take?

Then, around 1990, came the Human Genome Project. Sold to the Congress and public as an undertaking comparable in scale and significance to the Manhattan Project that produced the first atomic bomb, and directed by Francis Collins, now Director of the whole of NIH, the Human Genome Project (HGP) was a 13-year project coordinated by the US Department of Energy and the National Institutes of Health. During the early years of the HGP, the Wellcome Trust (UK) became a major partner; additional contributions came from Japan, France, Germany, China, and others. The project goals were to; identify all the approximately 20,000-25,000 genes in human DNA; determine the sequences of the 3 billion chemical base pairs that make up human DNA; store this information in databases; improve tools for data analysis; transfer related technologies to the private sector, and to address the ethical, legal, and social issues (ELSI) that may arise from the project. Note that nowhere 
in this list of aims was anything said about translating that information into new cures for human diseases, yet that was the chief raison d'etre given to Congress to justify its multi-billion dollar cost. The Human Genome Project was completed in 2003, but the National Human Genome Research Institute, which grew out of it, is still a major component of $\mathrm{NIH}$. Its mission statement is: "NHGRI's mission has evolved over the years to encompass a broad range of studies aimed at understanding the structure and function of the human genome and its role in health and disease. To that end, the institute supports the development of resources and technology that will accelerate genome research and its application to human health." Note the emphasis on human health, which is in part because Congress and the public are increasingly asking NHGRI officials where all the promised cures are.

Finally, let's look at the unprecedented doubling of the NIH budget, from about $\$ 13$ billion to about $\$ 26$ billion, which took place from 1998 to 2003. It was this, more than anything else, that led to NIH becoming the 500-lb gorilla in the scientific funding zoo. Selling that to the Congress and public required tactics that would have made Willy Loman proud. The primary argument was, as you can probably now guess, that doubling the budget would lead to faster cures for more diseases.

Well, here we are almost ten years after that, and members of Congress and their constituents are now starting to get more than just a little impatient. Where, they ask, are all these promised cures? What is being done with all that money you asked for to help translate scientific discovery into better health?

The right answer, of course, is that the cures will come, but that they take a long time and often come from directions that are not obvious at the moment. Cisplatinum was discovered by a microbial biochemist who was interested in seeing what would happen if dividing bacteria were placed in an electric field. Modern molecular biology and the whole of the biotechnology industry, from which many of these cures will certainly arise, has grown out of the discovery that bacteria make specific cuts in DNA as a means of telling self from nonself. The vast majority of disease treatments in use today can be traced back to work that had no disease-related objective whatsoever; that wasn't trying to translate into anything; that was motivated by nothing more than individual curiosity.

The right answer is also that it takes, on average, about 15 years and over $\$ 1$ billion to develop a drug; that for every successful pharmaceutical, more than 6,000 completely new compounds have to be invented; that most promising therapies fail, in Phase 2 clinical trials, because our animal models for diseases are not very good at predicting whether a treatment will work in people; that the success rate for small molecule drug and even biopharmaceutical development is so low that a batting average like that would get you drummed out of baseball in Little League.

Yet no one in authority seems comfortable giving those answers. Instead, to placate the public and its elected officials, the NIH is now proposing to create a new center, aimed specifically at advancing the translation of basic research into the clinic. Proposed just a few months ago by Francis Collins (http://feedback.nih.gov/index.php/ faq-ncats/\#one), the National Center for Advancing Translational Sciences (NCATS) has this as its stated scientific rationale:

Rapid progress in scientific research and the increased availability of innovative technologies have generated unprecedented potential for advancing the translation of basic discoveries into therapeutics. At the same time, the process of drug discovery remains a challenging and riskladen endeavor. These opportunities and challenges have prompted the National Institutes of Health to propose formation of a new Center focused on accelerating the development and delivery of new, more effective therapeutics. This proposed Center is envisioned to be a tremendous resource for the entire translational science community. It would develop and offer innovative services and expertise in moving promising products through the development pipeline, as well as develop novel approaches to therapeutics development, stimulate new avenues for basic scientific discovery, and complement the strengths of existing NIH research activities.

Approval of this Center is being rushed through. Why does it seem so important to get it done before the end of fiscal year 2012? I think one reason is the fear on the part of those pushing for it, that Barack Obama may be a one-term President, and that a rightwing Republican Administration might so slash federal spending as to make creation of such a Center, which would have an initial budget of at least $\$ 500$ million, politically impossible. But here is the rationale that is being given officially for the haste:

Every family that has ended up at the end of a medical odyssey only to learn that we do not yet have an effective treatment or cure knows why we are in a hurry. While we have learned an enormous amount about disease and health from our research investments, many diseases and conditions lack treatments. Dr. Collins, the NIH Director, asked a key advisory committee to look at how NIH could realign our resources to speed development of new interventions and they concluded that the scientific opportunities are here now. NIH feels compelled to move quickly to get this proposed new Center running so that it may accelerate the important translational work that is ongoing at NIH. 
What exactly would this proposed Center do? I think that's unclear given the information we have so far. Again, the official statement is focused on getting to faster cures:

The central role of the proposed Center would be to establish and provide focused, integrated, and systematic approaches for building new bridges that link basic discovery research with therapeutics development and clinical care. Translational sciences are increasingly becoming multi-sector endeavors involving industry, government, academia, and other sectors. Through the proposed Center, NIH would play a key role in convening these cross-sector collaborations to advance therapeutics development. The proposed Center also could ... focus research efforts in high-need areas that attract little commercial interest, such as rare and neglected diseases, and drug rescue and repurposing research. The functions and activities of the proposed Center would include:

"providing a visible, central locus for access to resources, tools, and expertise related to translational medicine;

"streamlining and improving the process of therapeutics development;

"serving as a catalyst, resource, and convener for collaborative interactions by supporting novel and innovative partnerships between multiple key stakeholders, including academia, government, industry, venture capitalists, and non-profit organizations;

"expanding the pre-competitive space by, among other things, enabling and providing incentives for greater sharing of scientific information and publication of negative results;

"supporting and strengthening translational medicine and therapeutics research, including providing access to services and resources for high-throughput screening, assay development, medicinal chemistry, and preclinical modeling;

"training translational research investigators; and

*enhancing communication among all stakeholders.

An obvious question is what sorts of therapeutics would be the focus of the proposed Center? From the available information, it sounds like the answer is everything but the kitchen sink: In addition to strengthening and streamlining the process of developing small-molecule compounds into drugs, the Center would support research aimed at accelerating the development of a full range of products and techniques for the diagnosis, treatment, and prevention of disease, including diagnostics, biologics, medical devices, and behavioral interventions. Okay, maybe it's everything 'including' the kitchen sink.

And this is where my worry comes in. Regardless of what the new Center actually does, the way it is being sold is the same as the way the War on Cancer and Human Genome Project and the NIH budget doubling were sold: just let us do this, and you'll see cure after cure emerge as if by magic. But the fact is, curing a disease is one of the hardest things that human beings have ever tried to do, and the most spectacular cures usually arise from work that is originally not aimed at trying to cure anything. All the targeted programs in the world aren't going to change that. What they do instead is to ramp up expectations that cannot possibly be fulfilled.

And we've seen, in the examples I've given and others I could cite, that that's exactly what has happened every time we have overpromised the curing of disease. You can see now why I worry that the NCATS might be a case of doing the same thing over and over again and expecting a different outcome. Which would be crazy, right?

But I don't think it has to be that way. I'm not opposed in principle to the idea of the NCATS. In fact, I'm intrigued by it. I think that, if the new Center is pitched properly and does certain things - and, more importantly, does not do certain things - it has a chance to make a very positive impact, not just on human health, but on the way medical research is perceived. Next month, I'll offer my suggestions for what those things might be.

In the meantime, I think it's vital that we all communicate to the powers that be the importance of continuing to support, and extol, fundamental, hypothesis-driven and discovery-oriented research that is motivated largely by curiosity. Because if we don't, in trying to give people what they say they want, there is great danger that not only will we make them increasingly frustrated because they aren't getting it, but also that we will stifle, through lack of funding, the creative leaps that are actually our best chance of finding it. Henry Ford put it best, I think: "If I had asked people what they wanted, they would have said faster horses."

Published: 31 January 2011

\footnotetext{
doi:10.1186/gb-2011-12-1-102

Cite this article as: Petsko GA: The long and the short of it. Genome Biology 2011, 12:102.
} 